dem Minimalverthe nähert, wobei Seitenentladung nicht mehr eintritt.

6. Der Grund zu den in \$. 6 und 7 erhaltenen Resultaten, sowie zu den Variationen in der Richtung und Grö/se des Ausschlages, welche bei sehr langen Disjunctionsfunken oorkommen, scheint in dem Umstande zu liegen, da/s die Entladung, wenn der Widerstand im Entladungsbogen (wie hier) klein ist, aus mehreren hin- und heryehenden Strömen (Oscillationen) besteht. Es beruht auf der Intensität der Entladung und auf der Länge des Funkens, in wie fern nur die erste oder auch die folgenden dieser Oscillationen Disjunctionsströme su Stande bringen.

7. Auch der Funken, welcher sich bei elehtrischer Entladung zwischen zwei Wassermengen zeigt, ist elektromotorisch.

11. Ueber die durch .letherschwingungen erregten .Hitschwingungen der Körpertheilchen und deren Kückwirkung auf die ersteren, besonders zur Erklärung der Dispersion und ihrer Anomalien; von It: Sellmeier.

(Fortsetzung von S. 421.)

\title{
§. 5.
}

Der Erfahrung zufolge zeigt ein natürlicher Lichtstrahl nie eine Polarisation, weder eine lineare, noch eine kreisförmige, noch eine elliptische. Dagegen lehrt die Theorie, dafs in einem homogenen Lichtstrahl, aus wie viel Theilen man ihn auch zusammensetzen möge, die Bahn, welche ein Aethertheilchen zu einer Zeit durchläuft, nothwendig eine Ellipse ist, wenn man mit diesem $\mathbf{W}$ orte auch den Kreis (beide Ellipsenaxen einander gleich) und die gerade 
Linie (kleine Axe gieich Null) umfafst; d. h. es kann ein solcher Strahl nicht anders gedacht werden, als zu jeder Zeit polarisirt, sey es elliptisch, oder kreisförmig, oder geradlinig. Um diesen scheinbaren Widerspruch zu heben, ist die Annahme nothwendig, dafs die elliptische Bahn des Aethertheilchens Veränderungen unterworfen ist, und zwar mit einer solchen Geschwindigkeit, dal's innerhalb jeder noch so kurzen bemerkbaren Zeit alle transversalen Schwingungsrichtungen gleich stark vertreten sind, und dafs in derselben kurzen Zeit die beiden Richtungen, in welchen das Aethertheilchen die Bahn durchlaufen kann, gleich oft vorkommen; es müssen nämlich in diesem Falle die in dem erwähnten Zeitraume nach einander vorkommenden Polarisationszustănde des Strahls im Gesichtseindrucke sich gegenseitig aufbeben und können daher nicht zur Wabrnehmung gelangen.

Andererseits haben Fizeau und Foucault im Sonnenlicht Interferenzen wahrnehmen können bei einem Gangunterschiede von fast 4000 Wellenlängen; ja später hat Fizeau im Natriumlicht noch bei einer Wegdifferenz von 50,000 Wellenlängen Interferenzen beobachtet. Dies beweist, dals jene Veränderungen der Bahnellipse des Aethertheilchens keine plötzlichen seyn können, sondern dafs sie, im Verhältnifs zur Geschwindigkeit der Schwingungen, sehr langsam und stetig erfolgen, mögen sie sich beziehen auf die Lage der Ellipsenaxen, oder auf deren absolute Gröfse und ihr Verhältnifs zu einander, oder auf die Richtung, in welcher das Aethertheilchen die Bahn durchläuft, diese Richtung dadurch wechselnd, dafs die kleine Axe durch den Werth Null hindurchgeht.

Zerlegt man nun einen natürlichen homogenen Lichtstrahl in zwei senkrecht auf einander polarisirte, bringt die letztern dann in eine einzige Polarisationsebene und lälst sie mit einander interferiren, so kann man aus dem vorhergehenden schliefsen, dafs innerhalb jeder noch so kurzen bemerkbaren Zeit alle möglichen Phasenunterschiede zwischen beiden Strahlen in gleichem Grade vertreton 
seyn, und dafs daher die in demselben kurzen Zeitraum nach einander folgenden Interferenzwirkungen in dem einheitlichen Lichteindrucke sich gegenseitig aufheben werden, oder dals, wie man es etwas uneigentlich auszudrücken pflegt, solche Strahlen niemals interferiren können, yanz so, wie es mit der Erfahrung übereinstimmt.

Gegenwärtig ist jedoch nur das Verhalten der Schwingungsamplitude in einem linear polarisirten Strahl für unsern Gegenstand von Interesse. In Bezug auf diese folgt ans obiger Eigenschaft des natürlichen Lichts:

Die Schwingungsamplitule in einem linearpolarisirten homogenen Lichtstrahl ist nicht constant, sondern veränderlich; sie ändert sich aber nicht plötzlich oder sprunguceise, sondern im Verhältni/s zur Geschwindigkeit der Schwingungen sehr langsam und continuirlich.

Auch sieht man ein, dal's die Amplitude zu Zeiten zum Worthe Null herabsinken und dals alsdann die Phase sich um eine halbe Undulation ändern wird. Für die mittlere Anzahl derjenigen Schwingungen, welche von zwei benachbarten Nullwerthen eingeschlossen sind, und welche wir eine Schwingungsreike nennen wollen, könnte man aus obigen Interferenzbeobachtungen eine untere Gränze bestimmen. Sollen nämlich die beiden $\mathrm{Z}$ weige eines Strahls bei ilurer Wiedervereinigung eine bemerkbare Interferenzwirkung hervorbringen, so müssen die zusammentallenden Schwingungen öfter einer und derselben, als zwei verschiedenen Schwingungsreihen angehören. Demnach mufs im Sonnenlicht eine Schwingungsreihe durchschnittlich mehr als 8000 , in Natriumlicht mehr als 100,000 Schwingungen enthalten. Indefs sind jedenfalls diese Zahlen viel zu klein, besonders die erstere; denn aus den bekannten Oscillationsdauern des Lichts folgt mit Evidenz, dafs wenn in einer Secunde eine Million Schwingungsreihen auf einander folgten, jede derselben doch durchschnittlich aus mehr als 400 Millionen Schwingungen bestehen würde. Aus der Gröfse dieser Zahlen läfst sich auch abnehemen, dal's es wohl eine vergebliche Mühe seyn würde, wenn man 
einen Strahl so schnell unterbrechen wollte, dafs dadurch der vorhin hervorgehobene Satz von der Allmäligkeit dei Amplitudenänderung seine Gültigkeit verlöre.

Da nun nach dem vorigen Paragraphen die Verschiebungen $\xi_{0}, \eta_{0}, \varsigma_{0}$ des Gleichgewichtsortes jedes Körpertheilchens der Verschiebung $\varrho^{\prime}$ des Aethers im linear polarisirten Strahl proportional sind, so müssen aúch die Schwingungen dieses Gleichgewichtsortes nach jeder der drei Schwingungsaxen aus eben solchen Schwingungsreihen bestehen, deren Amplitude, mit Null beginnend und endend, langsam und stetig zu- und abnimmt. Bei der Integration der Gleichung (2) wurde stillschweigend angenommell, dafs die Amplitude $a_{1}$ constant sey, und es ist daher zu untersuchen, welchen Einflufs ihre Veränderlichkeit auf die Integralgleichungen (3) und (4) ausübt.

Das Zu - und Abnehmen der Schwingungsamplitude des Aethers, also auch der des Gleichgewichtsortes des Körpertheilchens, kann man offenbar ansehen als erzeugt durch das successive Entstehen und Verschwinden vou aufeinander gelagerten gleichphasigen elementaren Schwingungsreihen, deren Amplitude unendlich klein, aber constant ist. Das Entstehen wie das Verschwinden jeder dieser elementaren Reihen erzeugt nun Schwingungen des Körpertheilchens, welche einer der Gleichungen (3) und (4) entsprechen müssen, und welche sich ebenfalls auf einander lagern.

Angenommen, es bestehe die Amplitude $a_{0}$ aus den zu den Zeiten $t_{1}, t_{2}, t_{3} \ldots$ entstandenen unendlich kleinen Elementen $d\left(a_{0}\right)_{1}, i\left(a_{0}\right)_{2} \ldots$, so dafs

$$
\xi_{0}=\left[d\left(a_{0}\right)_{1}+\lambda\left(a_{0}\right)_{2}+\ldots\right] \sin 2 \pi \frac{t+a}{t}
$$

ist. Dann sind, wenn die Schwingungsdauern $\delta$ und $r$ von einander abweichen, die $\mathrm{zu}$ denselben Zeiten entstehenden wesentlichen Schwingungen des Körpertheilchens folgende:

$\frac{\iota^{2}}{t^{2}-\gamma^{2}} d\left(a_{0}\right)_{1} \sin 2 \pi \frac{t+\alpha}{t}, \frac{t^{2}}{2^{2}-\delta^{2}} d\left(a_{10}\right)_{2} \sin 2 \pi \frac{t+\alpha}{\tau} \ldots$, 
Durch die Aufeinanderlagerung derselben entsteht die Summe

$$
\frac{\frac{1}{2}^{2}}{\tau^{2}-\delta^{2}} a_{0} \sin 2 \pi \frac{t+\alpha}{\tau},
$$

und man sieht also, dafs der Ausdruck für die wesentlichen Schwingungen der Gleichung (3) durch die Veränderlichkeit der Amplitude $a_{0}$ keinen Einflufs erleidet.

Sind dagegen die Schwingungsdauern $\delta$ und $t$ einander gleich, so ist die Summe der zu den Zeiten $t_{1}, t_{2} \ldots$ entstandenen wesentlichen Schwingungen des Körpertheilchens folgende:

$$
-\pi\left[\frac{t-t_{1}}{\delta} d\left(a_{0}\right)_{1}+\frac{t-t_{2}}{\delta} \partial\left(a_{0}\right)_{2}+\ldots\right] \cos 2 \pi \frac{t+\alpha}{\delta} .
$$

Bezeichnet man also die Amplitude der durch den vorstehenden Ausdruck dargestellten Schwingungen mit $a$, so muls dieselbe stets der Gleichung

genügen.

$$
\frac{d a}{d t}=\frac{\pi}{\delta} a_{0}
$$

Was endlich die nach einander entstehenden unwesentlichen Schwingungen des Körpertheilchens anbetrifft, so haben diese nicht, wie die wesentlichen, gleiche Phase; während bei den letztern der Werth von $\alpha$ überall derselbe, nämlich der in den Schwingungen des Gleichgewichtsorts vorkommende ist, durchläuft bei den unwesentlichen Schwingungen die Grölse $\beta$ alle zwischen 0 und $\delta$ liegenden Werthe, und zwar sind im Allgemeinen alle diese Werthe in gleichem Grade vertreten. Die Folge davon ist, dafs die aus der Aufeinanderlagerung dieser Schwingungen resultirende Gesammtamplitude nicht die Summe der unendlich kleinen Einzelamplituden ist, sondern dafs vielmehr das Quadrat der Gesammtamplitude gleich ist der Summe der Qundrate der Einzelamplituden; und daraus folgt, dafs die Gesammtamplitude immer unendlich klein bleibt. Wir wollen dies in Bezug auf die unweseutlichen Schwingungen der Gleichung (3), wo dies 
Resultat von besonderer Wichtigkeit ist, specieller ausführen.

Es gehe zur Zeit $t^{\prime}$ die Amplitude $a_{0}$ des Gleichgewichtsorts in $a_{0}+\Delta a_{0}$ über, und es werden dadurch die unwesentlichen Schwingungen

$$
\Delta b \sin 2 \pi \frac{t+\beta}{\delta}
$$

erzengt. Wenn also vor der Zeit $t^{\prime}$

$$
\xi=\frac{t^{2}}{t^{2}-\delta_{2}} a_{11} \sin 2 \pi \frac{t+\alpha}{t}+b \sin 2 \pi \frac{t+\beta^{\prime}}{\delta}
$$

war, so ist nach derselben

$$
\begin{aligned}
\xi=\frac{\tau^{2}}{\tau^{2}-\delta^{2}}\left(a_{0}+d a_{0}\right) \sin 2 \pi \frac{t+a}{r} & +b \sin 2 \pi \frac{t+\beta^{\prime}}{\delta} \\
& +d b \sin 2 \pi \frac{t+\beta}{\delta} .
\end{aligned}
$$

Zur Zeit $t^{\prime}$ kann $\xi$ nur Einen Werth haben, und es müssen daher in Bezug auf diese Zeit beide Ausdrücke einander gleich seyn. Daraus folgt

$$
\Delta b \sin 2 \pi \frac{t^{\prime}+\beta}{\gamma}=-\frac{\tau^{2}}{\tau^{2}-\gamma^{2}} \Delta a_{0} \sin 2 \pi \frac{t^{\prime}+a}{\tau} .
$$

Ebenso mufs die aus beiden Gleichungen bestimmte Geschwindigkeit $\frac{d \xi}{\partial t}$ für die Zeit $t^{\prime}$ dieselbe seyn, woraus folgt

$$
d b \cos 2 \pi \frac{t^{\prime}+\beta}{\delta}=-\frac{\delta}{\tau} \cdot \frac{t^{2}}{\tau^{2}-d^{2}}\left\langle a a_{0} \cos 2 \pi \frac{t^{\prime}+a}{r} .\right.
$$

Aus den letzten beiden Gleichungen ergiebt sich für den Werth von $\beta$

$$
\operatorname{tg} 2 \pi \frac{t^{\prime}+\beta}{\delta}=\frac{\tau}{\delta} \operatorname{tg} 2 \pi \frac{t^{\prime}+\alpha}{\tau},
$$

und für die Amplitude $\Delta b$

$$
\text { (b) } \Delta b^{2}=\left(\frac{\tau_{2}}{\tau^{2}-j^{2}} d a_{0}\right)^{2}\left(\sin ^{2} 2 \pi \frac{t^{\prime}+a}{\tau}+\frac{\delta^{2}}{\tau^{2}} \cos ^{2} 2 \pi \frac{t^{\prime}+a}{\tau}\right) \text {. }
$$

Hătte $a_{0}$ um $\Delta a_{0}$ ab- statt zugenommen, so würde man, $-\Delta a_{0}$ farr $\Delta a_{0}$ setzend, dieselben Werthe fur $\beta$ und $\Delta b$ erhalten haben.

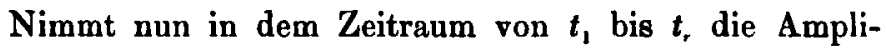
tude des Gleichgewichtsortes $r$ Mal um eine Gröfse $\Delta a_{0}$ 
zu oder ab, so werden dadurch $r$ unwesentliche Schwingungsreihen erzeugt, welche sich auf einander lagern, und indem man die daraus resultirenden Schwingungen durch

darstellt, hat man

$$
c \sin 2 \pi \frac{t+\%}{\pi}
$$

$c \sin 2 \pi \frac{t+\gamma}{\gamma}=\Delta b_{1} \sin 2 \pi \frac{t+\beta_{1}}{\gamma}+\Delta b_{2} \sin 2 \pi \frac{t+\beta_{2}}{\gamma}+\ldots$

$$
= \pm a b \sin 2 \pi \frac{t+\beta}{1},
$$

wo die Werthe von $\beta_{1}, \beta_{2} \ldots$ und die von $d b_{1}, d b_{2} \ldots$ aus den Gleichungen (a) und (b) zu entnehmen sind, indem man in denselben $t_{1}, t_{2} \ldots$ für $t^{\prime}$ setzt. $D a$ vorstehende Gleichung gültig seyn mul's für jeden Werth von $t$, der gröfser ist als $t$, so liefert sie folgende zwci Gleichungen :

$$
\begin{aligned}
& c \sin 2 \pi \frac{\gamma}{\gamma}=\Sigma a b \sin 2 \pi \frac{\beta}{\gamma}, \\
& c \cos 2 \pi \frac{\gamma}{\gamma}=\Sigma \lambda b \cos 2 \pi \frac{\beta}{\delta},
\end{aligned}
$$

und aus diesen folgt

$$
c^{2}=\Sigma a b^{2}+\sum d b_{m} \Delta b_{a} \cos 2 \pi \frac{\beta_{m}-\beta_{n}}{d},
$$

wo das zweite Glied auf der rechten Seite die Summe der $r(r-1)$ Producte bedeutet, welche man erhält, wenn man jeden der $r$ Werthe von $a b$ und dem zugehörigen $\beta$ in der angezeigten Weise mit jedem der $r-1$ andern Werthe von $d b$ und dem zugehörgen ; verbindet. Nimmt die Amplitude des Gleichgewichtsortes langsam und continuirlich zu oder ab, ist also die Zahl $r$ umendlich grols, dagegen der Werth von $d a_{0}$ unendlich klein, so wird es zu jedem dieser Producte viele andere absolut gleichwerthige geben, in denen aber der Werth von $2 \pi \frac{\beta_{m}}{\gamma}$ ebenso oft negativ als positiv ist. Die Summe dieser Producte ist daher gleich Null zu setzen, und die vorstehende Gleichung reducirt sicb auf

$$
c^{2}=\Sigma \Delta b^{2} \text {. }
$$


Bezeichnet man mit $d b^{\prime}$ den mittleren Werth $d b$, so hat man

$$
c^{2}=r . \Delta b^{\prime 2},
$$

also, da $A a$, und mithin auch $d b$ und $d b^{\prime}$ unendlich klein, dagegen $r$ unendlich grols ist,

$$
c^{2}=\infty \cdot \frac{1}{\infty^{2}}=\frac{1}{\infty} .
$$

Wenn daher die Schwingungsreihe des Gleichgewichtsortes mit der Amplitude Null beginnt und schliefst, und wenn diese Amplitude nur langsam und stetig sich ändert, so bleibt während der ganzen Schwingungsreihe die $\mathrm{Am}$ plitude der unwesentlichen Schwingungen unendlich klein.

Nur wem die beiden Schwingungsdauern $t$ und $i$ nur sehr wenig von einander verschieden sind, können, weil in diesem Falle einestheils nach (b) die Amplitude $A b$ sehr grofs ist im Vergleich zu $\Delta a_{0}$, anderntheils nach (a) der Werth von $\beta$ bei der Zunahme der Zeit $t^{\prime}$ nur langsam sich ändert, die sich auf einander lagernden unwesentlichen Schwingungen einen merklichen Amplitudenwerth erlangen und daher, weil die in ihnen enthaltenc lebendige Kraft für immer der Lichtbewegung verloren geht, einen merklichen Lichtverlust erzengen.

Durch diejenigen Lichtschwingungen, deren Dauer hin.

länglich abweicht von den eigenthiumlichen Schwingungs dauern eines Körpertheilchens, werden keine merklichen unwesentlichen Schwingungen des letztern erregt.

Es ist dieses Resultat, wie man sieht, eiuzig die Folge davon, dafs bei einem linear polarisirten homogenen Lichtstrabl die Amplitude des Aethers nur langsam und stetig sich ändert und jede $S$ chwingungsreihe mit Null beginnt und endet. Diese Eigenschaft polarisirter Strahlen ist daher von grofser Wichtigkeit: denn ohne sie würde in Folge der dann auftretenden, das Licht absorbirenden unwesentlichen Schwingungen der Körpertheilchen die Existens transparenter Körper kaum als möglich erscheinen.

Die Ursache dieser Eigenschaft sieht man übrigens leicht ein, wenn man auf die Entstehung des Lichtes zurtick- 
geht. Alles Licht, dies steht wohl unzweifelhaft fest, wird durch das Schwingen von Körpertheilchen erzeugt. Diese Schwingungen gehen nur sehr langsam an den Aether über, so dafs jedes Körpertheilchen, ähnlich einer Glocke oder Saite, nach jedem empfangenen Impuls eine lange Reihe von Aetherwellen auszusenden vermag, bevor es wieder zur Ruhe kommt. Empfängt, ehe letzteres der Fall ist, das Körpertheilchen einen neuen Impuls, so tritt dessen Wirkung zu den noch vorhandenen Wirkungen der vorhergehenden Impulse hinzu, und man sieht daber ein, dafs, wenn die Impulse sehr schnell auf einander folgen, jeder einzelne von ihnen die Amplitude und Phase der Schwingungen des Körpertheilchens nur sehr wenig verändern kann. Wenn daher bei sehr schneller Aufeinanderfolge der Impulse die Amplitude und Phase merkliche Aenderungen erleiden, so wird man diese als nabezu continuirlich ansehen können. Letzteres ist zwar nicht mehr der Fall, wenn die Impulse seltener sind; es giebt jedoch noch einen andern Umstand, der mit in Erwägung gezogen werden mufs. Ein Lichtstrahl, und wäre derselbe noch so dünn, ist stets das Erzeugnifs einer sehr grofsen Anzahl von Körpertheilen; das, was ein einzelnes dieser Theilchen zu demselben beitrïgt, ist verschwindend klein, um so mehr die Wirkung eines einzelnen Impulses, den dasselbe empfängt. Nun ist es möglich, dafs die Impulse, worin dieselben auch bestehen mögen, bald stärker, bald schwächer sind, und dais sie bald schneller, bald langsamer auf einander folgen. Auch können diejenigen Sch wingungen, welche mehr oder weniger einander parallel sind, bei den verschiedenen den Strahl erzeugenden Körpertheilchen bald mehr in gleichem, bald mehr in ungleichem Sinne geschehen. Endlich kann dies Alles, oder das Eine oder Andere, bald mehr in der einen, bald mehr in der andern Richtung der Fall seyn. In Folge dessen wird die Ellipse, welche an einer bestimmten Stelle des Lichtstrabls von einem Aethertheilchen durchlaufen wird, und welche das Resultat der Schwingungen aller jener Körper- 
theilchen ist, sich zwar ändern müssen, aber diese Aenderung wird nur eine langsame und continuirliche seyn können, was dann obige Eigenschaft des polarisirten Strahls zur Folge hat. Wenn, wie es vielleicht beim elektrischen Funken der Fall ist, die Körpertheilchen durch einen kräftigen Impuls urplötzlich in starke Schwingungen versetzt werden, so wird dies doch nie gleichzeitig geschehen; von denjenigen Körpertheilchen, welche Theil haben an der Erzeugung des Lichtstrahls, wird immer eines zuerst den Impuls empfangen, und die anderen werden allmählig nachfolgen. Die erwähnte Ellipse wird daher auch in diesem Falle, unendlich klein beginnend und endend, nur allmählig wachsen und wieder abnehmen können; wie es denn uberbaupt wohl keine natürlichen Ursachen oder künstlichen Mittel geben wird, durch welche bewirkt werden könnte, dafs die Aenderungen jener Ellipse sprungweise erfolgen.

\section{§. 6.}

Den Betrachtungen im vorigen Paragraphen zufolge können wir jetzt in den Schwingungsgleichungen eines Körpertheilchens das unwesentliche Glied fortlassen. Indem wir dann die Zeit $t$ mit einem positiven Durchgange des momentanen Gleichgewichtsortes durch den Ruheort anfangen lassen, fält auch die Constante $\alpha$ fort, so dafs die Schwingungsgleichung des momentanen Gleichgewichtsortes folgende ist.

$$
\xi_{0}=a_{0} \sin 2 \pi \frac{t}{\tau} .
$$

Die Verschiebung des Körpertheilchens in der Richtung der $x$ wird dann ausgedrückt

wenn die Schwingungsdauer $\tau$ des Lichts von der eigenthümlichen Schwingungsdauer $\delta$ des Körpertheilchens verschieden ist, durch die Gleichung

(5) $\quad \xi=\frac{\tau^{2}}{\tau^{2}-\delta^{2}} a_{0} \sin 2 \pi \frac{t}{\tau}-\frac{\tau^{3}}{\tau^{2}} \frac{\delta^{2}}{\xi_{0}}$;

Poggendorff's Annal. Bd. CXLV. 
wenn dagegen beide Schwingungsdauern einander gleich sind, durch die Gleichungen

$$
\left\{\begin{array}{l}
\xi=-a \cos 2 \pi \frac{t}{d}, \\
\frac{d a}{d t}=\frac{\pi}{d} a_{0} .
\end{array}\right.
$$

Wir wollen nun die durch diese Gleichungen dargestellten Schwingungen des Körpertheilchens näher ins Auge fassen und zugleich sehen, worin im Allgemeinen ihre Rückwirkung auf die Aetherschwingungen bestehen wird.

1. Die Gleichung (5) betreffend wollen wir zunächst bemerken, dals dieselbe sebr einfacb auf directem Wege sich herleiten lälst. Soll nämlich das Körpertheilchen um seinen Ruheort mit der Schwingungsdauer $\tau$ oscilliren, so mufs sich die beschleunigende Kraft $X$ ausdrücken durch die Gleichung

$$
X=-\frac{4 \pi^{2}}{z^{2}} \xi
$$

nach $(C)$ wird sie aber auch durch

$$
X=-4 a^{2}\left(\xi-\xi_{0}\right)
$$

ausgedrückt, und daraus folgt sofort

$$
\xi=\frac{x^{2}}{2^{2}-\delta^{2}} \xi_{0} \text {. }
$$

Aus der Gleichung (5) ergiebt sich nun Folgendes.

Die Schwingungsdauer des Körpertheilchens ist gleich der seines Gleichgewichtsortes, also aucb gleich der des Aethers. Daraus folgt:

Durch das Licht werden stets nur solche Schwingungen der Körpertheilchen erregt, welche mit den Lichtschwingungen selbst isochron sind.

Hinsichtlich der Phase lassen sich zwei wesentlich verschiedene Fälle unterscheiden, je nachdem $\delta$ kleiner oder gröfser ist als $\tau$. Im erstern Falle haben die Verschiebungen $\xi$ und $\xi_{0}$ gleiche, irn letztern entgegengesetzte Vorzeichen. 
Wenn also die eigenthümliche Schwingungsdauer des Körpertheilchens kleiner ist als die Schwingungsdauer seines momentanen Gleichgewichtsortes, so bewegen sich beide, das Körpertheilchen und sein Gleichgewichtsort, stets in gleicher Richlung, ist dagegen jene grö/ser als diese, so ist die Bewegnng des einen stets der des andern entgegengesetzt.

Im letztern Falle befinden sich daher beide, das Körpertheilchen und sein momentaner Gleichgewichtsort, stets auf entgegengesetzten Seiten des Ruheortes, in diesem sich jedesmal begegnend; im erstern dagegen sind sie stets auf derselben Seite, jedoch nie so, dafs sich das Körpertheilchen zwischen seinem momentanen Gleichgewichtsund seinem Rubeorte befindet, weil die Verschiebung $\xi$ nie kleiner seyn kann als $\xi_{0}$; es zeigt dies auch die aus (5) leicht hervorgehende Gleichung

$$
\frac{\partial^{2}}{\tau^{2}}=\frac{\xi-\xi_{0}}{\xi},
$$

nach welcher $\xi$ nicht kleiner als $\xi_{0}$ werden kann, ohne dafs eine der beiden Schwingungsdauern $\delta$ und $\boldsymbol{t}$ imaginair wird.

Die Amplitude des Körpertheilchens betreffend sieht man, dafs die Gleichung (5) dieselbe als unendlich grols ergiebt, wenn die beiden Schwingungsdauern $\delta$ und $\tau$ einander gleich sind, in welchem Falle jedoch die Gleichungen (6) zur Anwendung kommen. Entfernt sich $\delta$ von dem Werthe von $\tau$, sey es durch $\mathrm{Zu}$ - oder durch $\mathbf{A b}$ nahme, so verkleinert sich die Amplitude, und zwar zuerst sehr schnell, dann immer langsamer. Ist dann $\delta$ unendlich klein oder unendlich grol's geworden, so ist die Amplitude des Körpertheilchens im erstern Falle gleich der seines momentanen Gleichgewichtsortes, im letztern gleich Null.

Wenn die eigenthümliche Schwingungsdauer des Körpertheilchens unendlich klein ist, so befindet sich dasselbe stets in seinem momentanen Gleichgewichtsorte; 
ist sie unendlich gro/s, so verharrt das Körpertheilchen unbeweglich in seinem Ruheorte.

Es verdient nochmals hervorgeboben $\mathrm{zu}$ werden, dafs die wirkliche Schwingungsdauer eines Körpertheilchens keineswegs immer mit seiner eigenthümlichen übereinstimmt, dafs sie vielmehr in Folge von Oscillationen seines Gleichgewichtsortes jeden Werth haben kann, mag dieser noch so grofs oder noch so klein seyn; bewegen sich beide, das Körpertheilchen und sein Gleichgewichtsort, stets in gleicher Richtung, so ist die wirkliche Schwingungsdauer grö/ser als die eigenthïmliche, bewegon sie sich einander entgegengesetzt, so ist sie kleiner.

Was nun die Rückwirkung der dureh die Gleichung (5) dargestellten Schwingungen des Körpertheilchens auf die Aetherschwingungen anhetrifft, so mufs man beachten, dals die Amplitude desselben stets proportional ist der seines Gleichgewichtsortes, mithin auch der des Arthers. Wenn daher die Amplitude des Aethers am Ende einer Schwingungsreihe gleich Null geworden ist, so ist es auch die des Körpertheilchens. Letzteres behält also von der in seinen Schwingungen enthalten gewesenen lebendigen Kraft Nichts zurück, und dieselbe mufs daher der Lichtbewegung vollständig verblieben seyn, d. h. eine Absorption des lichts findet durch diese Schwingungen nicht statt. Dagegen leuchtet ein, dal's dieselben, da durch sie die Masse des Schwingenden vermehrt ist, einen Einflufs auf die Fortpflanzungsgeschwindigkeit des Licbts haben werden, d. h.

die durch die Gleichung (5) dargestellten Schwingungen der Körpertheilchen sind als die Ursache der Refraction anzusehen.

Es wird Diefs indefs weiter unten der Gegenstand besonderer und eingehender Betrachtungen seyn.

2. Indem wir zu den Gleichungen (6) übergehen, treten uns in denselben zwei die Phase und die Amplitude des Körpertheilchens betreffende Eigenthümlichkeiten entgegen. Die erste dieser Gleichungen enthält nämlich statt 
des in der Schwingungsgleichung des Gleichgewichtsortes vorkommenden Ausdrucks

den Ausdruck

$$
\sin 2 \pi \frac{t}{\delta}
$$

$$
-\cos 2 \pi \frac{t}{\delta}
$$

oder, wie man ibn auch schreiben kann,

Man ersieht daraus,

$$
\sin 2 \pi \frac{t-\frac{1}{4} d}{\delta} .
$$

da/s die Schwingungen des Körpertheilchens gegen die seines Gleichgewichtsortes, also auch gegen die des Aethers, um den vierten Theil einer Schwingungsdauer verspätet sind.

Die zweite Eigenthümlichkeit zeigt uns die zweite der Gleichungen (6). Man ersieht nämlich aus derselben unmittelbar,

da/s die Amplitude des Körpertheilchens während jeder Schioingung um die mit der Zahl $\pi$ multiplicirte Amplitude seines Gleichgewichtsortes zunimmt.

Hieraus erkennt man auch sofort, worin die Rückwirkung dieser Schwingungen auf die des Aethers bestehen wird. Dem Gesagten zufolge wächst nämlicb die Amplitude des Körpertheilchens so lange, als die Schwingungsreihe des Aethers dauert, und wenn letztere aufhört, hat jene gerade ihr Maximum erreicht. Die in der Schwingungsbewegung des Körpertheilchens enthaltene lebendige Kraft geht daher vollständig der Lichtbewegung verloren, d. h.

die durch die Gleichungen (6) dargestellten Schwingungen der Körpertheilchen sind die Ursache der Absorption.

Die Absorption besteht in einer Verkleinerung der Schwingungsamplitude des Aethers. Diese Verkleinerung setzt voraus,

dafs die Schwingungen des Körpertheilchens viederum Oscillationen des Aethers erregen, welche gegen die des 
erstern um den vierten Theil einer Schwingungsdauer verspätet sind.

Diese erregten Aetherschwingungen sind nämlich daun den erregenden gerade entgegengesetzt, und die algebraische Summe aus beiden hat daher den Erfolg einer Verkleinerung der erregenden Aetherschwingungen.

Hat die Schwingungsreihe in erregenden Lichte ihr Ende erreicht, d. h. ist ihre Amplitude bis zu Null herabgesunken, so werden die Oscillationen der Körpertheilchen, deren Amplitude jetzt gerade im Maximum sich befindet, noch fortdauern, und sie werden auch noch fortfahren, um eine Viertel-Undulation verspätete Aetherschwingungen zu erregen. Da diese aber nicht mehr in entgegengesetzte Aetherschwingungen aufgehen, weil solche nicht mehr vorhanden sind, so werden sie im Allgemeinen als ausgestrahltes Licht zum Vorschein kommen; es ist dies das Fluorescenzlicht, welches demnach jedesmal erst dann beginnt, wenn eine Schwingungsreihe im erregenden Lichte ihr Ende erreicht hat, was allerdings Millionen Male in der Secunde vorkommen mag.

Der Umstand, dafs die absorbirende und die ausstrahlende Thätigkeit der Körpertheilchen sich nur dadurch von einander unterseheiden, dafs die von denselben erregten Aetherschwingungen im erstern Falle die Schwächnng des der Absorption ausgesetzten Lichtes bewirken, im letztern aber als ausgestrahltes Licht zur Erscheinung kommen, kann als eine Begründung des von verschiedenen Physikern aufgestellten Satzes angesehen werden, dals das Absorptions - und das Ausstrablungsvermögen eines Körpers bei allen Schwingungsdauern einander proportional seyen.

Um die Einfachheit der vorstehend entwickelten Vorstellung von der absorbirenden und emittirenden Thätigkeit hier nicht zu stören, wollen wir die Besprechung einiger dieselbe betreffenden Besonderbeiten auf einen späteren Paragraphen verschieben.

3. Die durch die Gleichungen (5) und (6) dargestell- 
ten Bewegungen eines Körpertheilchens sind so wesentlich verschieden, dafs eine Betrachtung über den Uebergangsfall, wo die Differenz zwischen der eigenthümlichen Schwingungsdauer des Körpertheilchens und der Oscillationsdauer des Lichts sebr klein ist, nicht als überflüssig erscheinen dürfte. $Z u$ diesem $Z$ wecke haben wir von der allgemeinen Gleichung (3) auszugehen, nämlich

$$
\xi=\frac{\tau^{2}}{\tau^{2}-\delta^{2}} a_{0} \sin 2 \pi \frac{t}{\tau}+b \sin 2 \pi \frac{t+\beta}{\delta} .
$$

Bestimmt man das letzte Glied auf der rechten Seite durch den Anfangszustand, der bei $t=0$ stattfindet, indem man die Anfangsverschiebung mit $\xi$, die Anfangsgeschwindigkeit mit $\frac{d \Sigma^{\prime}}{d t}$ bezeichnet, und setzt man den so erhaltenen Werth des erwähnten Gliedes in die vorstehende Gleichung, so erhält man

$$
\begin{aligned}
\xi=\frac{\tau^{2}}{\tau^{2}-\delta^{2}} a_{0} \sin 2 \pi \frac{t}{\tau}-\frac{\delta}{\tau} \cdot \frac{x^{2}}{\tau^{2}-\delta^{2}} a_{0} \sin 2 \pi \frac{t}{\delta} \\
+\frac{\delta}{2 \pi} \cdot \frac{d \xi^{\prime}}{d t} \sin 2 \pi \frac{t}{\delta}+\xi \cos 2 \pi \frac{t}{\delta} .
\end{aligned}
$$

Dieser Gleichung mufs nun eine Form gegeben werden, bei welcher die Verschiebung $\boldsymbol{\xi}$ nicht unendlich grofs wird, wenn $\operatorname{man} t=\delta$ setzt; wir schreiben sie daher wie folgt;

$$
\begin{aligned}
& \xi=-2 \frac{\tau^{2}}{\tau^{2}-\delta^{2}} a_{0} \sin 2 \pi \frac{t(\tau-\delta)}{2 \tau \delta} \cos 2 \pi \frac{t(\tau+\delta)}{2 \tau \delta} \\
& \quad+\frac{\tau}{\tau+\delta} a_{0} \sin 2 \pi \frac{t}{\delta}+\frac{\delta}{2 \pi} \cdot \frac{d \xi^{\prime}}{d t} \sin 2 \pi \frac{t}{\delta}+\xi \cos 2 \pi \frac{t}{\delta}
\end{aligned}
$$

Hier stellt das erste Glied auf der rechten Seite Schwingungen dar mit der Oscillationsdauer $\frac{2 \imath \delta}{r+\delta}$, und mit der veränderlichen Amplitude

$$
2 \frac{\tau^{2}}{x^{2}-\delta^{2}} a_{0} \sin 2 \pi \frac{t(x-\delta)}{2 \pi \delta} .
$$

Die Periode dieser Veränderung beträgt $\frac{\tau \delta}{\tau-\delta}$, innerbalb welcher die Amplitude von 0 bis $2 \frac{x^{2}}{t^{2}-t^{2}}$ steigt und dann wieder bis 0 herabsinkt. Wenn also $\tau$ und $\delta$ nur sehr 
wenig verschieden sind, so ist diese Amplitude des Körpertheilchens zur Zeit ihres Maximums sehr grofs in Vergleich zu der des Gleichgewichtsortes, während die Amplitude des zweiten Gliedes nur ungefähr die Hälfte von der des Gleichgewichtsortes beträgt. Die beiden letzten Glieder betreffend läfst sich Folgendes bemerken. Wenn die durch die vorstehende Gleichung dargestellten Schwingungen zur Zeit $t=0$ ihre Entstehung haben, so kann man annehmen, dafs das Körpertheilchen vorher in Ruhe war; denn hatte dasselbe bereits eine Bewegung, so gehört dieselbe anderen Schwingungen an, welche man bei ihrer Fortdauer gesondert von den neu entstandenen und als sich auf diese auflagernd betrachten kann. Demnach kann man sowobl die Anfangsverschiebung $\xi^{\prime}$ als auch die Anfangsgeschwindigkeit $\frac{d \xi^{\prime}}{d t}$ als gleich Null ansehen. Jedenfalls darf man die durch die letzten drei Glieder der Gleichung dargestellten Schwingungen für so klein halten, dafs sie nur um die Zeit des Minimums der Amplitude des ersten Gliedes bemerkbar sind. Lälst man sie daher fort, so hat man

$$
\text { (7) } \xi=-2 \frac{\tau^{2}}{\tau^{2}-\delta^{2}} a_{0} \sin 2 \pi^{t} \stackrel{(\tau-\delta)}{2 \tau \delta} \cos 2 \pi \frac{t(r+\delta)}{2 \tau \delta} \text {. }
$$

Lälst man die Differenz $(\tau-\delta)$ unendlich klein werden, so wird die Periode $\frac{\tau \delta}{\tau-\delta}$ der Amplitudenänderung unendlich grofs, und die Amplitude wächst fortwährend, ohne jemals in endlicher Zeit unendlich grofs zu werden. Verwandelt man den Ausdruck

$$
\sin 2 \pi \frac{t(x-\delta)}{2 \pi \delta}
$$

nach der bekannten Formel

$$
\sin x=x-\frac{x^{3}}{6}+\ldots
$$

in eine Reihe, so besteht der Ausdruck für die Amplitude aus einer Anzahl von Gliedern; setzt man dann $\tau=\delta$, so verschwinden alle Glieder bis auf das erste, und es entsteht

$$
\xi=-\pi \frac{t}{\delta} a_{0} \cos 2 \pi \frac{t}{\delta},
$$


wofür man wegen der Veränderlichkeit der Amplitude $a_{0}$ die Gleichungen (6) setzen mufs. - Somit sind wir auf directem Wege von der Schwingungsweise, welche das Körpertheilchen befolgt, wenn die Schwingungsdauern $\tau$ und $\delta$ verschieden sind, zu der ganz anderen Schwingungsweise gelangt, welche stattfindet, wenn beide Schwingungsdauern einander gleich sind.

Um die durch die Gleichung (7) dargestellten Schwingungen genauer zu betrachten, kann man ihr folgende Gestalt geben:

$\xi=2 \frac{\tau^{2}}{\tau^{2}-\delta^{2}} a_{11} \sin 2 \pi \frac{t(2-\delta)}{2 \times \delta} \cdot \sin 2 \pi\left[\frac{t}{t}+\frac{t(2-\delta)}{2 \cdot \delta}-\frac{1}{4}\right]$.

In dieser Form stellt sie Schwingungen dar mit der Oscillationsdauer $r$, aber mit dem veränderlichen Phasenunterschiede $\left[\frac{t(x-\delta)}{2 \tau \delta}-\frac{1}{4}\right]$, während der Ausdruck für die Amplitude derselbe geblieben ist.

Es sey zuerst $t$ gröfser als $\delta$. Wie man sieht, ist dann zu Anfange der Zeit $t$ die Amplitude gleich Null, und der Phasenunterschied $=-\frac{1}{4}$, d. b. die Schwingungen des Körpertheilchens sind gegen die des Aethers um $\frac{1}{4}$ Undulation zurück. Darauf nimmt der absolute Werth des Phasenunterschiedes ab und die Amplitude zu. Bei $t=\frac{1}{2} \frac{\tau \delta}{\tau-\delta}$ hat die letztere ihr Maximum $2 \frac{\tau^{2}}{\tau^{2}-t^{2}} a_{0}$ erreicht, und der Phasenuntersehied ist gleich Null, d. h. Aether und Körpertbeilchen bewegen sich jetzt in gleicher Richtung. Hierauf wird der Phasenunterschied positiv und wächst, während die Amplitude wieder abnimmt. Bei $t=\frac{t \delta}{\tau-\delta}$ ist diese gleich Null und jener $=+\frac{1}{4}$, d. $h$. die Schwingungen des Körpertheilchens sind denen des Aethers um $\frac{1}{4}$ Undulation voraus. Nimmt die Zeit $t$ noch weiter zu, so ändert die Amplitude das Zeichen; um dieselbe noch ferner als positiv zu betrachten, muls man die Phase um eine halbe Undulation ändern. Dadurch wird 
der Phasenuntersehied $=\frac{1}{4}-\frac{1}{2}=-\frac{1}{4}$, und man sieht also, dafs derselbe plötzlich von $+\frac{1}{4}$ auf $-\frac{1}{4}$ zurückspringt, und dafs daher nun derselbe Gang auf's Neue beginnt. Die Dauer der Periode sowohl der Pbasen -, als auch der Amplitudenänderung ist also gleich $\frac{t \delta}{i-\delta}$, und der Phasenunterschied durchläuft während derselben die Werthe

$$
-\frac{1}{4} \ldots 0 \ldots+\frac{1}{4} \text {. }
$$

Ist $\tau$ kleiner als $\delta$, so geht die Gleichung in Folgende über:

$\xi=2 \frac{\tau^{2}}{\delta^{2}-\tau^{2}} a_{0} \sin 2 \pi \frac{t(\lambda-\tau)}{2 \tau \delta} \cdot \sin 2 \pi\left[\frac{t}{\tau}-\frac{t(\delta-\tau)}{2 \tau \delta}-\frac{1}{4}\right]$.

Aus dieser Gleichung ist leicht zu ersehen, dafs der Gang der Erscheinung dem vorigen ganz ähnlich ist und sich von demselben nur dadurch unterscheidet, dafs jetzt während der Periode, deren Dauer $\frac{\tau \delta}{\delta-\imath}$ beträgt, der Phasenunterschied die Werthe

$$
-\frac{1}{4} \ldots \frac{1}{2} \ldots+\frac{1}{4}
$$

durchläuft, und dafs daher, wenn die Periode zur Hälfte abgelaufen ist und die Amplitude ibren Maximalwerth $2 \frac{\tau^{2}}{d^{2}-r^{2}} a_{0}$ erreicht hat, Aether und Körpertheilchen nicht in gleicher Richtung, sondern einander entgegengesetzt sich bewegen.

Was nun die Rückwirkung dieser Schwingungen auf die des Aethers anbetrifft, so finden sich hier die Wirkungen der durch die Gleichungen (5) und (6) dargestellten Schwingungen vereinigt. Man sieht leicht ein, dafs um die Mitte der Periode der Einflufs auf die Wellenlänge vorherrschend ist, dafs derselbe aber desto mehr zurücktritt und dafür der Einflufs auf die Amplitude des Aethers sich geltend macht, je mehr man sich dem Anfange oder Ende der Periode nähert. In der ersten Hälfte der Periode geht ein Theil der lebendigen Kraft vom Aether an das Körpertheilchen über: derselbe wird aber in der zweiten Hälfte an den Aether vollständig zurückgegeben, 
vorausgesetzt, dals die Amplitude des Aethers im ankommenden Lichtstrahl während der Dauer der Periode sich nicht geändert hat. Ist aber letateres der Fall - und nach dem vorigen Paragraphen ist es dies allerdings, und zwar um so mehr, je länger die Periode dauert, d. h. je kleiner die Differenz zwischen $\tau$ und $\delta$ ist - , so geht dem Aether ein Theil der lebendigen Kraft für immer verloren, d. h. es findet eine wirkliche Absorption des Lichtes statt, welche demnach um so gröfser ist, je mehr die Differenz zwischen den Schwingungsdauern $\tau$ und $\delta$ der Null sich nähert.

Diese Nebenabsorption, welche stattfindet bei nicht vollkommener Uebereinstimmung der Schwingungsdauern $\tau$ und $\delta$, hat also ihren Grund in der Veränderlichkeit der Ellipse, welche die Aethertheilchen in einem natürlichen Lichtstrahl durchlaufen.

\section{§. 7.}

Es dürfte nicht ohne Interesse seyn, zu sehen, wie man die im vorigen Paragraphen entwickelten Sätze experimentell veranschaulichen kann. Als das geeignetste Mittel hierzu erscheint das Fadenpendel. Der Gleichgewichtsort der Pendelkugel befindet sich stets vertical unter dem Aufhängepunkte; um also zu bewirken, dafs der erstere in horizontalen Schwingungen sich befinde, braucht man nur den letztern in eben solche Schwingungen zu versetzen. Hierzu dürfte am besten ein Pendel mit unbiegsamer Stange dienen, so dafs dieses die Rolle des Aethers zu spielen hat, während die Kugeln der Fadenpendel die Körpertheilchen vorstellen. Der Schwierigkeit, mittels eines Pendels einen Punct in horizontaler gerader Linie zu bewegen, kann mun dadurch ausweichen, dals man hier, wo es nur auf den allgemeinen Charakter der Erscheinung ankommt, mit folgender sehr einfacher Einrichtung sich begnügt.

Die Stange des Aetherpendels ist an ihrem obern Ende unter rechtem Winkel fest verbunden mit einer hinreichend 
langen horizontalen Stange, welche an jedem Ende mittels einer Schneide oder verticalen Spitze auf einer Unterlage ruht, so dafs die Verbindungslinie der beiden Schneiden die Drehaxe des Aetherpendels ausmacht. An der horizontalen Stange befinden sich mehrere der Pendelstange parallele Arme von verschiedener Länge, an deren unterem Ende die Vorrichtung zum Aufhängen der Fadenpendel sich befindet. Wenn also das Aetherpendel in seiner Ruhelage ist, so hefinden sich die Auf hängepuncte der Fadenpendel vertical unter der Drehaxe, und wenn jenes Schwingungen macht, so bewegen sich diese in einem Bogen, welcher, wenn er nur kurz ist, als gerade horizontale Linie angesehen werden kann. Um Aenderungen der Schwingungsebene zu verhindern, kann man jeden Pendelfaden, auf welchem die Pendelkugel etwa mittels eines Häkchens gleitet, mit seinen Enden an zwei benachbarte Aufhängepuncte befestigen, welche ron der Drehaxe gleichweit entfernt sind. Es ist nothwendig, den Apparat so einzurichten, dafs man mehrere Fadenpendel gleichzeitig aufhängen kann, und dafs die Möglichkeit gewährt ist, die Armlänge, d. h. die Entfernung der Aufhängepuncte von der Drehaxe, je nach Bedürfnils, bald grölser, bald kleiner zu nehmen.

Es sey $l^{\prime}$ die Länge des Aetherpendels, $q$ seine Neigung zu der durch die Drehaxe gehenden Verticalebene, $\xi^{\prime}$ der Abstand seines Schwingungspunctes ron dieser Ebene, $\tau$ seine Schwingungsdauer, $g$ die Schwerkraft, so ist die auf den Schwingungspunct wirkende beschleunigende Kraft

$$
\frac{\partial^{2}(l \varphi)}{\partial t^{2}}=-g \sin \varphi=-\frac{g}{l} \xi^{\prime},
$$

also deren zur erwähnten Ebene senkrechte Componente

$$
\frac{\partial^{2} \xi^{\prime}}{\partial t^{2}}=-\frac{g}{l^{\prime}} \xi^{\prime} \cos \varphi \text {. }
$$

Wird die Amplitude $a^{\prime}$ (das Maximum von $\xi^{\prime}$ ) so klein genommen, dals man immer $\cos \varphi=1$ setzen kann, und schreibt man $\frac{4 \pi^{2}}{\tau^{2}}$ für $\frac{g}{l^{\prime}}$, so entsteht 
und dies giebt

$$
\frac{d^{2} \xi^{\prime}}{d t^{2}}=-\frac{4 \pi^{2}}{\tau^{2}} \xi^{\prime}
$$

$$
\xi^{\prime}=a^{\prime} \sin 2 \pi \frac{t+\alpha}{\tau},
$$

wo $\alpha$ eine Constante ist. Was hier $\xi^{\prime}$ und $a^{\prime}$ ist, werde in Bezug auf den Aufhängepunkt des Fadenpendels mit $\xi_{0}$ und $a_{0}$ bezeichnet, dann wird also die Bewegung dieses Aufhängepunktes in Folge der Schwingungen des Aetherpendels dargestellt durch die Gleichung

$$
\xi_{0}=a_{0} \sin 2 \pi \frac{t+\alpha}{r} .
$$

Es sey endlich $\delta$ die eigenthümliche Schwingungsdauer des Fadenpendels und $\xi$ der Abstand seiner Kugel von der durch die Drebaxe gebenden Verticalebene, so hat man, wenn man wieder den Cosinus des Ausschlagswinkels gleich Eins setzt,

$$
\frac{d^{2} \xi}{d t^{2}}=-\frac{4 \pi^{2}}{\delta_{2}}\left(\xi-\xi_{0}\right)=-\frac{4 \pi^{2}}{\delta^{2}}\left(\xi-a_{0} \sin 2 \pi^{t+a}{ }_{t}^{-\alpha}\right) .
$$

Dies ist aber eben die Differentialgleichung (2), woraus folgt, dafs unser Apparat innerbalb der Gränzen, in denen man den Cosinus des Ausschlagswinkels gleich Eins setzen darf, recht wohl geeignet ist, die aus der Gleichung (2) hergeleiteten Bewegungsgesetze darzustellen. Die Kleinheit der Ausschläge ist selbstverständlich besonders da eine nothwendige Bedingung, wo es auf die Gleichheit von $\delta$ und $\tau$ ankommt.

Das Fadenpendel mit beweglichem Aufhängepunkte kann man bezeichnen als Absorptionspendel, wenn seine Schwingungsdauer mit der des Aetherpendels vollkommen übereinstimmt, als Refractionspendel, wenn beide Schwingungsdauern beträchtlich, und als Uebergangspendel, wenn sie nur wenig von einauder abweichen. Bei jedem dieser Pendel kann man seine Aufmerksamkeit auf $Z$ weierlei richten, bämlich zuerst auf die Art seiner Bewegung, wie sie durch die Schwingungen des Aetherpendels bervorgerufen wird, und sodann auf die Rückwirkung jener auf 
diese. Im erstern Falle ist es vortheilhaft, die Pendelkugel so klein zu nehmen, dals die Rückwirkung auf die Amplitude oder Schwingungsdauer des Aetherpendels unmerklich ist, im letztern, wo diese Rückwirkung besonders hervortreten soll, ist eine gröfsere Kugel nothwendig, dessen Gewicht dem des Aetherpendels angenähert gleichist oder dasselbe übersteigt.

1. Das Absorptionspendel. Bei Anwendung einer sehr lileinen Kugel zeigt der Apparat, dafs, wenn man das A therpendel in Schwingungen setzt, die dann entstehenden Schwingungen des Fadenpendels gegen jene um $\frac{1}{4}$ Undulation verspätet sind, so wie dafs deren Amplitude fortwährend zunimint, und zwar während jeder Schwingung un $\pi a_{0}$, unter $a_{0}$ die Amplitude des Aufhängepunktes verstanden.

Wendet man eine grofse Kugel an, so sieht man, dafs, während die Amplitude des Absorptionspendels wächst, die des Aetherpendels abnimmt, bis diese gleich Null geworden ist und jene ihr Maximum erreicht hat; ferner siebt man, dals darauf das Aetherpendel wieder anfängt zu schwingen (das Fluorescenzlicht darstellend), während die Schwingungen des Absorptionspendels wieder abnehmen, dafs aber jetzt die Schwingungen des Aetherpendels gegen die des andern nicht mehr um ${ }_{4}^{1}$ Undulation voraus sind, sondern um ebenso viel zurück. - Es mufs hier auf einen Unterschied aufmerksam gemacht werden, der zwischen den Erscheinungen, welche dieser Apparat darbietet, und denen des Lichts stattfindet. Beim Apparat geht die ganze lebendige Kraft des Aetherpendels nach und nach an ein und dasselbe Absorptionspendel über; eine Aetherwelle dagegen giebt, weil sie im Raume fortschreitet, ibre lebendige Kraft nach und nach an immer neue absorbirende Körpertheilchen ab, und jedes der letzteren erbält von jeder neu ankommenden Welle einen neuen Antheil, so lange die Schwingungsreihe dauert. Ferner, wenn die Schwingungsreihe zu Ende ist und nun das Körpertheilchen den Aether in Bewegung setzt, so 
erzeugt jede seiner Schwingungen eine neue Aetherwelle, welche gleich ihren Vorgängern und Nachfolgern im Raume sich ausbreitet und in der Ferne verliert; bei unserem Apparat dagegen geht die ganze Bewegung des Absorptionspendels nach und nach an ein und dasselbe Aetherpendel über und häuft sich hier an, um dann von Neuem an das Absorptionspendel überzugehen. So wechseln beide Pendel in ihrer Bewegung mit einander ab; wenn das eine zur momentanen Ruhe (oder zum Minimum seiner Bewegung) kommt, befindet sich jedesınal das andere im Maximum seiner Schwingungen, und dieses Spiel setzt sich so lange fort, als die Bewegung überhaupt dauert (oder bis sie sehr klein geworden ist). Jedesmal, wenn das Absorptionspendel Bewegung von dem Aetherpendel empfängt, stellt es die Rolle der absorbirenden, jedesmal, wenn es solche an das Aetherpendel abgiebt, die Rolle der emittirenden Thätigkeit des Körpertheilchens dar. Um die Dauer der Periode zu bestimmen, während welcher das Eine oder das Andere geschieht, seyen $a^{\prime}, a, a_{0}$ die Amplituden des Aetherpendels, des Absorptionspendels und des Aufhängepunktes des letzteren, $l^{\prime}$ und $l_{0}$ die Pendellänge und die Armlänge des Aufhängepunktes; dann ist nach dem Bisherigen

$$
\frac{\partial a}{\partial t} \tau=\pi a_{0}=\pi \frac{l_{0}}{l} a^{\prime}
$$

und wegen des Princips der Erhaltung der Kraft

$$
m a^{2}+m^{\prime} a^{\prime 2}=k,
$$

wo $k$ eine Constante, $n$ die Kugelmasse des Absorptionspendels, und $m^{\prime}$ eine von der Masse des Aetherpendels und ihrer Vertheilung abhängige Grölse ist. Aus der letztern Gleichung folgt

$$
a^{\prime}=\sqrt{\frac{k-m a^{2}}{m^{\prime}}},
$$

und hiermit giebt die erstere

$$
\frac{\partial a}{\sqrt{k-m a^{2}}}=\pi \frac{l_{0}}{l^{\prime} V^{\prime} \overline{m^{\prime}}} \frac{\partial t}{\tau} \text {. }
$$


Die Integration liefert

$$
\frac{t}{\tau}=\frac{l^{\prime}}{\pi l_{0}} \sqrt{\frac{m^{\prime}}{m}} \cdot \operatorname{arc} \operatorname{tg} V \frac{m a^{2}}{m^{\prime} a^{12}}+\text { Const. }
$$

Das Integral ist für die Daner der Absorption von $a=0$ bis $a^{\prime}=0$, und für die Dauer der Emission von $a^{\prime}=0$ bis $a=0$ zu nehmen. Bezeichnet man also mit $T$ die erstere, mit $\boldsymbol{T}^{*}$ die letztere Iauer, so hat man schliefslich

$$
\frac{T}{\tau}=\frac{T^{\prime}}{c}=\frac{1}{2} \frac{l^{\prime}}{l_{0}} V \frac{m^{\prime}}{m} .
$$

An dem Apparat wird man demnach noch zeigen können, dal's die beiden Zeiten, in welchen die Bewegung von den Aether- auf das Absorptionspendel und dann von dem letztern wieder auf das erstere übergeht, einander gleich sind (die Gleichheit des Absorptions - und Emissionsvermögens darstellend); dals diese Zeiten unabhängig sind von den Maximal-Amplituden der Pendel (darstellend die Unabhängigkeit des Absorptionsvermögens von der Intensität des Lichts), und dafs sie umgekehrt proportional sind einestheils der Armlänge, anderntheils der Quadratwurzel aus dem Kugelgewicht des Absorptionspendels.

Man darf bei den Versuchen nicht übersehen, dal's in den nicht $2 u$ vermeidenden unwesentlichen Scluwingungen, in dem Umstande, dafs die Bahnen der Aufhängepunkte Bogen sind statt horizontale Linien, in der Veränderlichkeit der Scbwingungsdauer des Aetherpendels, wenn das Fadenpendel mit demselben gleichgerichtet oder entgegengesetzt schwingt oder in Ruhe ist, in der Reibung und dem Luftwiderstande, sowie in sonstigen Unvollkommenheiten des Apparates Ursachen genug gegeben sind, welche in den Erscheinungen, welche der Apparat darbieteu soll, Störungen bewirken. So sieht man gewőhnlich, dafs das Absorptionspendel, wenn es zur momentanen Ruhe kommen sollte, statt dessen kleine mit dem Aetherpendel gleichgerichtete Schwingungen macht, dafs diese Schwingungen nach jeder Periode grölser erscheinen, dafs dann auch das Aetherpendel nicht mebr ganz zur Ruhe 
kommt, und dafs schliefslich, wenn die Gesammtbeweguug schon sehr geschwächt ist, die Schwingungen beider Pendel fortwährend gleichgerichtet sind. Abgesehen von solchen Störungen der Erscheinungen, die auch bei den folgendeu Versuchen auftreten, wird der Apparat recht wohl geeignet seyn, seinen $Z_{w e c k}$ zu erfüllen.

2. Das Refractionspendel. Zunächst mufs bemerkt werden, dafs dieser Name ein sehr uneigentlicher ist. Beim Licht wird die Refraction dadurch erzeugt, dafs die Körpertheilchen, deren eigenthümliche Schwingungsdauer nicht mit der Oscillationsdauer des Aethers übereinstimnt, durch ibre Mitschwingungen eine Aenderung der Fortpflanzungsgeschwindigkeit oder, was hier dasselbe ist, der Wellenlänge bewirken, während die schon bei der Entstehung der Wellen gegebene Schwingungsdauer nicht geändert werden kann. Bei unserm Apparat dagegen, wo von einer Fortpflanzung der Schwingungen des Aetherpendels nicht die Rede seyn kann, wird durch die Mitschwingungen des Refractionspendels statt der Wellenlänge die Schwingungsdauer geändert und diese Aenderung der Schwingungsdauer repräsentirt also die Aenderung der Wellenlänge beim Licht, und damit auch die Refraction.

Bei Anwendung sehr kleiner Kugeln kann man sogleich zwei Refractionspendel aufhängen, von denen das eine für sich allein eine beträchtlich kürzere, das andere eine betrăchtlich lăngere Schwingungsdauer hat, als das Aetherpendel. Setzt man dann das Aetherpendel in Schwingungen, so sieht man, dafs die Bewegungen der Refractionspendel sehr unregelmäfsig sind. Es rührt dies daher, dafs sich mit den wesentlichen Schwingungen noch die unwesentlichen mischen. Um die letzteren nicht zur Existenz kommen zu lassen, muf's die Schwingungsamplitude des Aetherpendels, mit Null anfangend, sehr langsam und stetig wachsen und ebenso wieder abnehmen. Als Mittel hierzu bietet sich in vortrefflicher Weise das Absorptionspendel dar. Wie wir gesehen haben, geht die Schwingungshe-

Poggendorff's Annal. Bd. CXLV. 
wegung eines solchen allmählig an das Aetherpendel über und ebenso allmählig an das Absorptionspendel zurück, und Beides geschieht um so langsamer, je kleiner die Armlänge des letztern ist. Die so erzeugten Schwingungen des Aetherpendels sind ganz ähnlich einer Schwingungsreihe, die ein durch Zerlegung geradlinig schwingendes Aethertheilchen ausführt, nur jedenfalls viel regelmälsiger. Fügt man also zu den beiden Refractions- noch ein $\mathbf{A b -}$ sorptionspendel mit sehr kleiner Armlänge, und setzt man dann das letztere in Bewegung, so sieht man, dafs die erstere jetzt in der That nur oder fast nur die wesentlichen Schwingungen machen, dafs diese mit den Schwingungen des Aetherpendels gleichdauerig sind, und dafs sie mit den letztern gleichzeitig von Null bis zum Maximum wachsen und dann bis Null wieder abnehmen. Ferner beobachtet man, dal's die Kugel des kürzeren Refractionspendels mit dem Aetherpendel stets gleichgerichtet schwingt, und zwar mit der Amplitude $\frac{z^{2}}{x^{2}-d^{2}} a_{0}$, dafs dagegen die Schwingungen, welche die Kugel des längern macht, denen des Aetherpendels entgegengesetzt sind und die Amplitude $\frac{\boldsymbol{r}^{2}}{j^{2}-\iota^{2}} a_{0}$ haben. Diese letztern Beobachtungen lassen sich auch am Apparate leicht erklären. Denkt man sich nämlich durch die Kugel des ruhenden Refractionspendels eine verticale Linie und in derselben oberhalb der Kugel einen Punct, der von der letztern um die Länge $\boldsymbol{l}^{\prime}$ eines mit dem Aetherpendel isochron schwingenden Fadenpendels entfernt ist, so mufs dieser Punct, da das Refractionspendel mit dem Aetherpendel eben isochron schwingen soll, fest seyn, d. h. der Pendelfaden (oder die Ebene der beiden Fäden des Pendels) mufs stets durch diesen Punct gehen oder nach demselben gerichtet seyn. $\mathrm{Da}$ nun bei dem längeren Refractionspendel dieser Punct unterhalb des Authängepunctes (oder der beiden Aufhängepuncte) liegt, so müssen Kugel und Aufhängepunct sich stets in entgegengesetzter Richtung bewegen; und da die Amplituden Beider sich verhalten, wie ibre Entfernungen 
rom festen Puncte, so hat man, wenn $l$ die Länge des Refractionspendels, $a$ die Amplitude seiner Kugel bedeuten,

also

$$
a: a_{0}=l^{\prime}: l-l^{\prime},
$$

$$
a=\frac{l}{l-l^{\prime}} a_{0}=\frac{\tau^{2}}{\jmath^{2}-\tau_{2}} a_{0} .
$$

Bei dem küzern Refractionspendel liegt der feste Punct oberhalb des Aufbängepunctes, dieser und die Kugel bewegen sich daher stets in gleicher Richtung, und man findet auf dieselbe $W$ eise

$$
a=\frac{l^{\prime}}{l^{\prime}-l} a_{0}=\frac{\tau^{2}}{\tau^{2}-\delta^{2}} a_{0} .
$$

Dafs das Refractionspendel mit gro/ser Kugel einen Einflufs auf die Schwingungsdauer des Aetherpendels haben mufs, sieht man ebenfalls leicht ein. Die Spannung des Pendelfadens strebt nämlich den Aufhängepunct um die Drebaxe zu bewegen, wodurch die das Aetherpendel nach seiner Rubelage treibende Kraft vermehrt oder vermindert wird. Im erstern Falle wird die Schwingungsdauer verkleinert (eine Vergröfserung der Wellenlänge des Lichts darstellend), im letztern vergrölsert (entsprechend einer Verkleinerung der Wellenlänge). Hiernach sieht man leicht, dafs bei unserm Apparate eine Verlängerung der Schwingungsdauer nur stattfindet, wenn der vorhin erwähnte feste Punct zwischen dem Aufhängepuncte und der Drehaxe liegt; liegt derselbe unterhalb des Aufhängepunctes oder oberhalb der Drehaxe, so wird die Schwingungsdauer verringert. Wäre der Apparat von der Beschaffenheit, dafs der Aufhängepunct in gerader horizontaler Linie sich bewegte, so würde der Sinn der Wirkung auf die Schwingungsdauer blols davon abbängen, ob der feste Punct unter- oder oberhalb des Aufhängepunctes läge, d. h. ob $\delta$ gröfser oder kleiner wäre als $\tau$. Um nun die Aenderung der Schwingungsdauer an dem Apparat sichtbar zu machen, kann man die regelmälsigen Schwingungen eines Refractionspendels mit schwerer Kugel entbehren, bei denen die uuwesentlichen Schwingungen sich schwer ver- 
meiden lassen. Man kann statt dessen ein Fadenpendel mit schwerer oder sehr schwerer Kugel und von ungefäbr gleicher Schwingungsdauer mit der des Aetherpendels an einen der längern Arme hängen, dasselbe dann zugleich nit dem Aetherpendel aus der verticalen Lage entfernen, sey es in gleichem oder ungleichem Sinne, und dann beide freilassen. Man beobachtet dann Folgendes: Bewegen sich zu einer Zeit beide Pendel in gleichem Sinne, so kann der Ausschlagswinkel des Fadenpendels gröl'ser oder kleiner seyn, als der des Aetherpendels; im ersten Falle ist die Schwingungsdauer des letztern Pendels vergröfsert, im letzten verkleinert. Bewegen sich aber beide Pendel in entgegengesetzter Richtung, so ist die Schwingungsdauer stets verkleinert.

3. Das Uebergangspendel. Bei Anwendung einer sehr kleinen Kugel kann man beobachten, dals die Schwingungen des Fadenpendels zuerst sehr klein und um $\frac{1}{4}$ Lndulation verspätet sind, dal's die Amplitude wächst, während die Verspätung ab-oder zunimmt, je nachdem $\delta$ kleiner oder grölser ist als $\tau$, dafs die Schwingungen ihr Maximum erreicht haben, wenn sie mit denen des Aetherpendels gleich oder entgegengesetzt gerichtet sind, dafs sie dann wieder abnehmen und endlich verschwinden, wenn sie den Schwingungen des Aetherpendels um ${ }_{i}^{1}$ Undulation voraus sind, und dal's nun dasselbe Spiel von Neuem beginnt.

Mit schwerer Kugel bietet das Uebergangspendel Erscheinungen dar, deren Gang wegen der Aenderungen der Schwingungsdauer des Aetherpendels oft sehr complicirt ist. Wir können hier anf deren Betrachtung verzichten.

Von mehr Interesse für unsern Gegenstand ist der experimentelle Nachweis, dafs eine Lichtabsorption auch dann schon stattfindet, wenn die Schwingungsdauer des Aethers um ein Geringes grölser oder kleiner ist, als die eigenthümliche des Körpertheilchens. Zu diesem Zwecke mufs man die Natur wieder dadurch nachahmen, dafs man mittels eines Absorptionspendels eine Schwingungsreihe 
des Aetherpendels mit continuirlich zu- und abnehmender Amplitude erzeugt. Dazu ist aber erforderlich, dafs die Schwingungsdauer des letztern durch das Uebergangspendel keine merklichen Aenderungen erfabre, und dafs daher dessen Kugel sehr klein sey. Setzt man, nachdem diese Bedingung erfüllt ist, das Absorptionspendel in Bewegung, so sieht man, dafs die Schwingungen des Uebergangspendels weder denen eines Refractionspendels mit beträchtlich abweichender Schwingungsdauer völlig gleichen, weil die unwesentlichen Schwingungen nicht ganz ausbleiben, noch denen, welche wir vorhin am Uebergangspendel beobachtet haben, weil der Gewinn in der ersten Hălfte der Periode und der Verlust in der zweiten sich nicht völlig ausgleichen. Wenn das Aetherpendel nach Beendigung der Schwingungsreihe zur Ruhe kommt, so befindet sich das Uebergangspendel noch in Bewegung, und die in der letztern enthaltene lebendige Kraft stellt eben den Verlust dar, welchen das Licht während der Schwingungsreihe erlitten hat.

Neu-Britz bei Berlin, d. 18. Januar 1872.

(Der zweite Theil mit Nächstem.)

\section{Ueber Abscheidung krystallisirler Kieselsäure aus wäfsrigen Lösungen; von $O$. Maschke.}

M. eine ersten Versuche über diesen Gegenstand datiren aus dem Jahre 1855; sie wurden in der Zeitschrift der deutschen geologischen Gesellschaft veröffentlicht.

Im Interesse der Geologie habe ich diese Untersuchungen - allerdings mit oft grofsen Unterbrechungen bis jetzt fortgefübrt. Wäbrend dieser Zeit gelangten die 